Dom. Cien., ISSN: 2477-8818

Vol. 4, núm. 1, enero, 2018, pp. 44-64

Biodisponibilidad relativa del fósforo en fosfatos comerciales utilizados en la alimentación de pollos de engorde

\title{
Biodisponibilidad relativa del fósforo en fosfatos comerciales utilizados en la alimentación de pollos de engorde
}

\author{
Relative bioavailability of phosphorus in commercial phosphates used \\ in broilers
}

\section{Biodisponibilidade relativa de fósforo em fosfatos comerciais utilizados na alimentação de frangos de corte}

Marcelo Gil-Araujo I
jgilmarcelo@yahoo.com
Hirwin Rincon-Reyes II
hirwinsegundorincon@yahoo.com

\author{
Denisse M. Cedeño-Cantos ${ }^{\text {III }}$ \\ dcedenovet@hotmail.com \\ Carlos F. Mendoza-Vélez IV \\ nanfavi0507@hotmail.com \\ Janeth R. Jácome-Gómez V \\ jjacomeregion4@gmail.com
}

Recibido: 10 de septiembre de $2017 *$ Corregido: 28 de noviembre de $2017 *$ Aceptado: 27 de diciembre de 2017

I. Médico Veterinario, Universidad del Zulia, Maracaibo, Venezuela.

II. Médico Veterinario, Universidad del Zulia, Maracaibo, Venezuela.

III. Magíster en Procesamiento de Alimentos, Médico Veterinario Zootecnista, Docente de la Universidad Laica Eloy Alfaro de Manabí, Manta, Ecuador.

IV. Ingeniero Agropecuario Especialidad Pecuaria, Docente Instituto Tecnológico Superior Calazacon, Santo Domingo, Ecuador.

v. Magister en Producción Animal, Ingeniera Agropecuaria, Docente de la Universidad Laica Eloy Alfaro de Manabí, El Carmen, Ecuador. 


\section{Resumen}

El objetivo fue evaluar la biodisponibilidad relativa (BR) del fósforo $(\mathrm{P})$ contenido en fosfatos comerciales, utilizados en la alimentación de pollos de engorde (PE) a los 18 y 28 d de vida, 960 PE machos de la línea Ross, de 1 día de edad en 48 corrales de 20 PE cada uno, el diseño factorial 4x3 aleatorizado con 4 repeticiones. Cuatro fuentes dietéticas de $\mathrm{P}$ dos monocálcico (A y C), dicálcico (B) y tricálcico (D) en tres niveles de inclusión de $\mathrm{P}(0,15 \%, 0,25 \%$ y $0,35 \%)$. Los PE recibieron dietas isocalóricas, isoprotéicas e isocalcicas, y manejados bajo condiciones de tipo comercial, alojados en un galpón tradicional. Se analizó el peso corporal (PC), porcentaje de ceniza ósea de tibia (PCOT) y porcentaje de mortalidad (PM) a dos edades (18 y 28 d). Los análisis estadísticos realizados fueron las pruebas de ANOVA, correlación y regresión. En los resultados no se observó diferencias $(\mathrm{P}>0.05)$ entre fuentes de P en el PC y PM de los PE a los 18 y 28 días de edad. Se detectó diferencia estadística significativa $(\mathrm{P}<0,001)$ en los valores de $\mathrm{PC}$ por los niveles de inclusión de $\mathrm{P}(0,15 \%=530,94 \mathrm{~g}$; $0,35 \%=581,88 \mathrm{~g} \mathrm{y} 0,25 \%=582,63 \mathrm{~g}$ ). El PCOT por fuente de P presento diferencia estadística significativa $(\mathrm{P}<0,05)$ de monocálcico A (47,79ab mg\%), C (49,37a mg\%), dicálcico B (50,12a mg\%) y tricálcico D (45,10b mg\%) a los $18 \mathrm{~d}$. A los $28 \mathrm{~d}$, PC y PCOT se hallaron correlacionados ( $\mathrm{r}=0,584$; $\mathrm{P}<0,0001)$. En conclusión, las fuentes de fosfato evaluadas no tuvieron efectos sobre el $\mathrm{PC}$, pero presento diferencia para PCOT a los 18 y 28 días de edad. Sin embargo, el nivel de $0,15 \%$ en las fuentes de fosfato con menores PC y PCOT. La fuente B de P dicálcico a $0,35 \%$ obtuvo la mejor BR a los $28 \mathrm{~d}$ de edad PE en el PC y PCOT.

Palabras claves: pollos; biodisponibilidad; fosforo; peso; ceniza ósea.

\section{Abstract}

The objective was to evaluate the relative bioavailability (BR) of phosphorus (P) contained in commercial phosphates, used in the feed of broilers (EP) at 18 and $28 \mathrm{~d}$ of life, 960 PE males of the Ross line, of 1 day of age in 48 pens of $20 \mathrm{PE}$ each, the randomized $4 \times 3$ factorial design with 4 repetitions. Four dietary sources of $\mathrm{P}$ two monocalcic (A and C), dicalcic (B) and tricalcic (D) at three levels of $\mathrm{P}$ inclusion $(0.15 \%, 0.25 \%$ and $0.35 \%)$. The PE received isocaloric, isoproteic and isocalcic diets, and managed under commercial conditions, housed in a traditional shed. The body weight (WC), percentage of tibial bone ash (PCOT) and percentage of mortality (PM) at two ages (18 and $28 \mathrm{~d}$ ) were analyzed. The statistical analyzes performed were the ANOVA, correlation and regression tests. 
In the results, no differences were observed $(\mathrm{P}>0.05)$ between sources of $\mathrm{P}$ in the $\mathrm{PC}$ and $\mathrm{PM}$ of the $\mathrm{PE}$ at 18 and 28 days of age. Significant statistical difference $(\mathrm{P}<0.001)$ was detected in $\mathrm{PC}$ values by $\mathrm{P}$ inclusion levels $(0.15 \%=530.94 \mathrm{~g}, 0.35 \%=581.88 \mathrm{~g}$ and $0.25 \%=582.63 \mathrm{~g})$. The PCOT per source of $\mathrm{P}$ showed statistically significant difference $(\mathrm{P}<0.05)$ of monocalcic A $(47.79 \mathrm{ab} \mathrm{mg} \%), \mathrm{C}$ (49.37a mg\%), dicalcic B (50.12a mg\%) and tricálcico D ( 45.10b mg\%) at $18 \mathrm{~d}$. At $28 \mathrm{~d}, \mathrm{PC}$ and PCOT were correlated $(\mathrm{r}=0.584, \mathrm{P}<0.0001)$. In conclusion, the phosphate sources evaluated had no effect on PC, but presented a difference for PCOT at 18 and 28 days of age. However, the level of $0.15 \%$ in the phosphate sources with lower PC and PCOT. Source B of dicalcium P at $0.35 \%$ obtained the best BR at $28 \mathrm{~d}$ of age PE in the PC and PCOT.

Keywords: chickens; bioavailability; match; weight; bone ash.

\section{Resumo}

O objetivo foi avaliar a biodisponibilidade relativa (FB) de fósforo (P) contida em fosfatos comerciais, utilizados na alimentação de frangos de corte (EP) a 18 e 28 d de vida, 960 machos de PE da linha Ross, de 1 dia da idade em 48 canetas de 20 PE cada, o formato fatorial 4x3 aleatorizado com 4 repetições. Quatro fontes dietéticas de $\mathrm{P}$ dois monocálcicos (A e C), dicalcic (B) e tricalcic (D) em três níveis de inclusão de $\mathrm{P}(0,15 \%, 0,25 \%$ e $0,35 \%)$. O PE recebeu dietas isocalóricas, isoproteicas e isocácicas e administradas sob condições comerciais, alojadas em um galpão tradicional. O peso corporal (WC), a porcentagem de cinza de osso tibial (PCOT) e porcentagem de mortalidade (PM) em duas idades (18 e 28 d) foram analisados. As análises estatísticas realizadas foram os testes ANOVA, correlação e regressão. Nos resultados, não foram observadas diferenças $(\mathrm{P}>0,05)$ entre fontes de P no PC e PM do PE aos 18 e 28 dias de idade. Foi detectada diferença estatística significativa $(\mathrm{P}<0,001)$ em valores de $\mathrm{PC}$ por níveis de inclusão de $\mathrm{P}(0,15 \%=530,94 \mathrm{~g}, 0,35 \%=$ $581,88 \mathrm{~g}$ e $0,25 \%=582,63 \mathrm{~g})$. O PCOT por fonte de $\mathrm{P}$ apresentou diferença estatisticamente significante $(\mathrm{P}<0,05)$ de monocálcico A (47,79 mg mg\%), C (49,37a mg\%), Dicalcic B (50,12a mg\%) e Tricálcico D (45,10b mg\%) às $18 \mathrm{~d}$. Aos 28 dias, PC e PCOT foram correlacionados ( $\mathrm{r}=0,584, \mathrm{P}$ $<0,0001)$. Em conclusão, as fontes de fosfato avaliadas não tiveram efeito no PC, mas apresentaram diferença para PCOT aos 18 e 28 dias de idade. No entanto, o nível de 0,15\% nas fontes de fosfato com PC e PCOT inferiores. A fonte B de dicalcium P a 0,35\% obteve o melhor BR a 28 d de idade PE no PC e PCOT.

Palavras chave: galinhas; biodisponibilidade; fósforo; peso cinza óssea. 


\section{Introducción}

El fósforo (P) es un macromineral (Hemati, et al., 2013) y es componente esencial de las estructuras óseas del esqueleto (Rath, et al., 2000), interviene en los fosfolípidos, procesos de energéticos y material genético. La disponibilidad biológica del P de origen orgánico vegetal el $80 \%$ en forma fitato (Askelson, et al., 2014; Davies y Motzok, 1972; Kozlowski, et al., 2009), P no fitato y P inorgánico. El aporte de $\mathrm{P}$ inorgánico y $\mathrm{P}$ no fitato en las dietas convencionales cubren los requerimientos mínimos de este mineral (Coon, et al., 2007; Hughes, et al., 2009). Las fuentes más comunes de P grado alimenticio para la industria avícola son: fosfato monocálcico, fosfato dicálcico, fosfato tricálcico y roca fosfórica (Potchanakorn y Potter, 1987). En la fisiología intestinal del P, se encuentra la fosfatasa alcalina intestinal permite la absorción de Ca y P derivado de la materia orgánica e inorgánica (Gabel y Thomas, 1976; Galvanovskii, et al., 1985; Kunitz, 1960; Valinietse, et al., 1985) y por acción de la vitamina D (Peterlik y Wasserman, 1980).

La optima utilización de estas fuentes inorgánicas de $\mathrm{P}$, estarán limitadas por el conocimiento que se obtenga sobre el origen, procesamiento, composición y calidad del P seleccionado (Godoy, et al., 2007). Estos parámetros son englobados generalmente con el término de respuesta biológica o biodisponibilidad relativa (BR) del P. Numerosos trabajos han sido realizados sobre la BR de diferentes fuentes de P orgánico e inorgánico para la alimentación de las aves (Akpe, et al., 1987; Burnell, et al., 1990; Coon, et al., 2007; Fernandes, et al., 1999; Kim, et al., 2008; Martinez Amezcua, et al., 2004; Potter, et al., 1995; Ravindran, et al., 1995; Steinsberger, et al., 1987; Waibel, et al., 1984).

La BR del P (BRP) se ha estudiado en diferentes investigaciones por medio del peso corporal (PC) (Hussein, et al., 1993; Lima, et al., 1997), porcentaje de ceniza ósea de tibia (PCOT) (Burnell, et al., 1990; Gillis, et al., 1954; Godoy y Chicco, 1999; Lima, et al., 1997; Potchanakorn y Potter, 1987; Potter, et al., 1995), porcentaje de ceniza del dedo medio (Ravindran, et al., 1995) y porcentaje de mortalidad (PM) (Fernandes, et al., 1999). En este sentido el presente estudio tiene como objetivo evaluar la BRP en PC, PCOT y PM presente en cuatro fuentes de fosfatos comerciales utilizados en la alimentación para pollos de engorde (PE) a 18 y 28 días de vida. 


\section{Materiales y métodos}

Se utilizaron 960 PE de la línea Ross x Ross, machos, de un día de nacidos, vacunados contra Marek, Gumboro y Newcastle y tres revacunaciones (d 1, 7 y 15) en el agua de bebida. El manejo general de las aves fue similar a las explotaciones avícolas comerciales de la zona.

Las aves fueron distribuidas en 48 corrales metálicos ( $0.6 \mathrm{~m}$ de altura, 2 x $1.5 \mathrm{~m}$ ó $3 \mathrm{~m} 2)$ a una densidad de 7 aves/m2 (20 aves/corral), con comederos y bebederos de recepción hasta los diez (10) días de iniciado el experimento, bebederos de campana y comederos tubulares hasta el final del ensayo.

Las dietas tiene cuatro fuentes de fosfato, el P monocálcico (biosfos y monómero), dicálcico (fosbic) y tricálcico (tricalfos) se mezcló con la dieta basal y con tres niveles de inclusión de P $(0,15,0,25$ y $0,35 \%$ ) resultaron 12 dietas experimentales. El suministro del alimento fue tres veces diarias hasta el día 15 de vida y 2 veces (mañana y tarde) hasta el d 28 de edad y se determinó el consumo real por corral. La composición de la dieta basal para pollos de engorde (TABLA I). Las dietas fueron isoprotéicas, isoenergéticas e isocalcicas, según los requerimientos nutricionales para pollos de engorde según NRC (N.R.C., 1994) y la guía de manejo de la línea.

Tabla I. Composición porcentual de las dietas experimentales

\begin{tabular}{lcccc}
\hline \multirow{2}{*}{ Ingredientes } & $\%$ & \multicolumn{3}{c}{ Niveles de P } \\
\cline { 3 - 5 } & & $0,15 \%$ & $0,25 \%$ & $0,35 \%$ \\
\hline Maíz & 58 & - & - & - \\
Soya (48\% PC) & 33 & - & - & - \\
Aceite de soya & 5,7 & - & - & - \\
Sal & 0,3 & - & - & - \\
Metionina & 0,3 & - & - & - \\
Carbonato & 1,9 & - & - & - \\
Minerales1 y vitaminas2 & 0,8 & - & - & - \\
Monofosfato & - & 0,81 & 1,35 & 1,89
\end{tabular}




$\begin{array}{lcccc}\text { Dicálcico } & - & 0,66 & 1,10 & 1,54 \\ \text { Tricálcico } & - & 0,82 & 1,36 & 191 \\ \text { PCE3 , \% } & 22 & 22 & 22 & 22 \\ \text { EM, kcal/ kg } & 3100 & 3100 & 3100 & 3100 \\ \text { P total, \% } & 0,24 & 0,39 & 0,49 & 0,59\end{array}$

1Niveles de fósforos adicionados a la dieta basal. 2Vitaminas (por kg. De alimento): Vitamina A 4.000 U.I., Vitamina D 200 U.I: Riboflavina 3 mg, Ácido pantoténico 5 mg, Niacina 20 mg, Colina 450 mg, Vitamina B12 mg, Vitamina E 2 mg, Micro minerales: Mn 65mg, I 1 mg, Cu 8 mg Zn 50 mg, Fe 25 mg, Mg 500mg. 3PCE: proteína cruda estimada (Nx6.25). Fosfato monocálcico (A) = Biosfos comercial. Fosfato dicálcico $(\mathrm{B})=$ Fosbic comercial. Fosfato monocálcico $(\mathrm{C})=$ Monómero. Fosfato tricálcico defluorinado $(\mathrm{D})=$ Tricalfos comercial.

Fuente: Elaboración propia

Para determinar el PC medio se pesaron las 20 aves por corral, para cada tratamiento con sus respectivas replicas a los d 1, 7, 14, 18, 21 y 28 de iniciado el ensayo y a la misma hora (Potter, et al., 1995). Para obtener el PCOT, se sacrificaron cuatro aves por corral a los 18 y $28 \mathrm{~d}$ de edad para cada fuente y nivel de fosfato (S.A.S., 2013), se cumplió con la normativa legal de eutanasia de animales vigente y evitando el sufrimiento. Posteriormente, se procedió a la extracción completa de los miembros posteriores derecho e izquierdo, estos fueron identificados y almacenados en congelación a $-20^{\circ} \mathrm{C}$. El procesamiento de las tibias, se realizó según la metodología establecida (Fick, et al., 1979; Godoy y Chicco, 1999; Lima, et al., 1997), hasta obtener el peso de la ceniza ósea para cada hueso y expresarlo como porcentajes de ceniza ósea libre de grasa (A.O.A.C., 1984). La mortalidad se registró diariamente para cada corral/tratamiento, y posteriormente se determinó el PM acumulado tanto a el d 18 y 28 de edad (Gillis, et al., 1954; Godoy y Chicco, 1999).

Los análisis estadísticos fueron ejecutados por medio del paquete estadísticos SAS (S.A.S., 2014). Se un diseño factorial $4 \times 3$ aleatorizado con 12 tratamientos y 4 repeticiones (20 aves/repetición). Los tratamientos fueron cuatro fuentes de fosfatos (fosfato monocálcico $\mathrm{A}$ = biosfos; fosfato dicálcico $\mathrm{B}$ 
fosbic; fosfato monocálcico $\mathrm{C}=$ monómero y fosfato tricálcico $\mathrm{D}=$ tricalfos) y tres niveles de inclusión de P $(0,15 ; 0,25$ y $0,35 \%)$. Las variables dependientes fueron PC, PCOT y PM; las variables independientes fueron los tratamientos, fuente de fosfato, nivel de $\mathrm{P}$ y edad de beneficio.

Los datos se procesaron por medio del procedimiento de ANOVA (Proc GLM) (S.A.S., 2010), una vez detectada diferencia estadística, se realizó la prueba de comparación múltiple de diferencia de mínimos cuadrados medios (LSMEAN con la opción PDIFF). Se efectuó análisis de correlación de Pearson de las variables (Proc Corr) (S.A.S., 2010). Para todos los procedimientos, la significancia estadística fue preestablecida a P<0,05. La BRP de las fuentes para PC y PCOT se llevó a cabo por medio de la proporción relativo al intercepto de la regresión del patrón de fosfato monocálcico A, asignándose el valor del 100\% de BRP para su comparación con el resto de las fuentes en forma proporcional (Godoy y Chicco, 1999). La prueba de BRP de la pendiente asume una respuesta lineal de las variables y un intercepto común para las líneas de regresión (Littell, et al., 1997).

\section{Resultados y discusión}

Los resultados iniciales del PC de las aves a los 18 y $28 \mathrm{~d}$ de vida alimentados con dietas de las fuentes de fosfato no se detectó diferencia estadística significativa ( $\mathrm{P}>0,05)$, bajo este punto de vista de las fuentes de fosfato las necesidades nutricionales de los PE fueron cubiertas. Cuyos PC por fuente a los 18 y 28 d fueron el monocálcico A $(568,17 \pm 7,19$ g y 1234,25 18,64 g), dicálcico B $(562,5 \pm 9,36$ g y $1230 \pm 24,86$ g), monocálcico C $(575 \pm 4,58$ g y $1243,42 \pm 9,62$ g) y tricálcico D $(554,92 \pm 13,34$ g y $1200,33 \pm 33,67 \mathrm{~g}$ ), respectivamente. Estos resultados de diferentes fuentes de fosfato de los PC a los 18 y 28 d de edad de los PE no fueron consistentes con los resultados reportados en otras investigaciones (Godoy, et al., 2007; Godoy y Chicco, 2001; Lima, et al., 1997).

Los resultados del análisis de los niveles de inclusión de $\mathrm{P}$ en las dietas experimentales de los PE a los 18 y 28 d de edad, se observó significancia estadística $(\mathrm{P}<0,0001)$ con el nivel de $0,25 \%$ $(582,63 \pm 3,13 \mathrm{~g}$ y $1260 \pm 8,75 \mathrm{~g})$ y $0,35 \%(581,88 \pm 3,68 \mathrm{~g}$ y $1282,44 \pm 6,56 \mathrm{~g})$ de $\mathrm{P}$ fueron los mayores PC y el nivel de P de 0,15\% (530,94 $\pm 7,52 \mathrm{~g}$ y $1138,56 \pm 18,42 \mathrm{~g})$ fueron los menores PC, respectivamente. Esto resultados fueron soportados por los hallazgos de los trabajos de otros autores (Lima, et al., 1997; Potchanakorn y Potter, 1987); con los niveles bajos de inclusión de P en la dieta, se presentaron PC inferiores y a medida que se incrementó el porcentaje de $\mathrm{P}$ en la dieta, aumentó el 
PC, hasta cierto límite biodisponibilidad y fisiológico de la salud y necesidades nutricionales de las aves (Hussein, et al., 1990; Muir, et al., 1990).

Los resultados del análisis de los valores medios del PC de los PE, obtenidos del efecto de los 12 tratamientos a los 18 y 28 d de edad (TABLA II), donde el tratamiento 3 (fosfato monocálcico A y $0,35 \%$ de $\mathrm{P})$ mejoró significativamente $(\mathrm{P}<0,001)$ el $\mathrm{PC}$ arrojando el valor más alto $(589,5 \pm 0,87 \mathrm{~g}$ y $1.297,5 \pm 6,66 \mathrm{~g}$ ) y el tratamiento 10 (fosfato tricálcico D y $0,15 \%$ de P) el valor más bajo del PC $(495,75 \pm 9,59 \mathrm{~g}$ y $1.049,75 \pm 18,27 \mathrm{~g})$ a los 18 y $28 \mathrm{~d}$ de edad de los PE, respectivamente.

Tabla II. Efecto de la interacción de la fuente de $\mathrm{P}$ y concentración de $\mathrm{P}$ sobre el peso de los pollos a 18 y 28 días de vida. Valores expresados en media \pm error estándar. ANOVA

\begin{tabular}{|c|c|c|c|c|}
\hline \multirow{2}{*}{$\begin{array}{l}\text { Tratamiento } \\
\qquad(\mathrm{n}=48 \\
\text { corrales })\end{array}$} & \multirow{2}{*}{ Fosfato } & \multirow{2}{*}{ P (\%) } & \multicolumn{2}{|c|}{ Peso (g) [20 aves/corral] } \\
\hline & & & 18 (Días) & 28 (Días) \\
\hline 1 & & 0,15 & $535,75 \pm 2,66 b$ & $1152,75 \pm 3,15 d$ \\
\hline 2 & Monocálcico (A) & 0,25 & $579,25 \pm 4,19 a$ & $1252,25 \pm 0 b c$ \\
\hline 3 & & 0,35 & $589,5 \pm 0,87 \mathrm{a} * *$ & $1297,75 \pm 6,66 \mathrm{a}^{* *}$ \\
\hline 4 & & 0,15 & $523,75 \pm 10,79 b$ & $1120 \pm 20,67 d$ \\
\hline 5 & Dicálcico (B) & 0,25 & $582,5 \pm 5,98 \mathrm{a}$ & $1272,5 \pm 13,97 \mathrm{abc}$ \\
\hline 6 & & 0,35 & $581,25 \pm 7,76 a$ & $1297,5 \pm 4,92 \mathrm{a}$ \\
\hline 7 & & 0,15 & $568,5 \pm 2,53 a$ & $1231,75 \pm 17,49 \mathrm{c}$ \\
\hline 8 & Monocálcico (C) & 0,25 & $583,75 \pm 7,87 \mathrm{a}$ & $1239,5 \pm 16,95 \mathrm{bc}$ \\
\hline 9 & & 0,35 & $572,75 \pm 10,99 a$ & $1259 \pm 17,14 \mathrm{abc}$ \\
\hline 10 & & 0,15 & $495,75 \pm 9,59 c$ & $1049,75 \pm 18,27 \mathrm{e}$ \\
\hline 11 & Tricálcico (D) & 0,25 & $585 \pm 8,65 \mathrm{a}$ & $1275,75 \pm 25,56 \mathrm{ab}$ \\
\hline 12 & & 0,35 & $584 \pm 6,39 a$ & $1275,5 \pm 12,04 \mathrm{abc}$ \\
\hline
\end{tabular}


Fosfato monocálcico $(\mathrm{A})=$ Biosfos comercial. Fosfato dicálcico $(\mathrm{B})=$ Fosbic comercial. Fosfato monocálcico $(\mathrm{C})$ = Monómero. Fosfato tricálcico defluorinado $(\mathrm{D})=$ Tricalfos comercial. $(\mathrm{a}, \mathrm{b}, \mathrm{c})(\mathrm{a}$, b, c, d, e) Diferencia estatística significativa. $* *(\mathrm{P}<0,01)$.

Fuente: Elaboración propia

Asimismo, el efecto de las dietas experimentales sobre el PC de los PE, a los 18 d de edad estadísticamente diferentes $(\mathrm{P}=0,001)$ conformaron tres grupos de tratamiento: 1$)$ tratamientos $2,3,5$, 6, 7, 8, 9, 11 y 12 de PC elevado; 2) los tratamientos 1 y4 4 de PC moderado; y 3) tratamiento 10 de PC deficiente. Mientras que en el caso de las dietas experimentales suministradas a las aves por los PC a los $28 \mathrm{~d}$ de edad fueron estadísticamente diferentes $(\mathrm{P}=0,001)$ conformaron grupos de tratamiento: 1) tratamientos 3, 5, 6, 9, 11 y 12 de PC elevado; 2) tratamiento 7 de PC moderado elevado; 3) tratamiento 1 y 4 de PC moderado deficiente; y 4) tratamiento 10 de PC deficiente.

Estos resultados del efecto de las dietas de fosfatos monocálcicas, dicálcicos, tricálcicas con niveles de 0,25 y 35\% de P sobre PC a los 18 y 28 d de edad de los PE fueron superiores en ambas edades al cumplir con los requerimientos nutricionales de aves resultan controversiales con los resultados de otros autores, donde los PC más altos fueron de dietas con fosfatos dicálcicos (Arias, et al., 2008; Godoy y Chicco, 2005). Asimismo, estos resultados de las dietas con niveles bajos de inclusión de fosfatos tricálcicos producen PC más bajos a los 18 y 28 d de edad en los PE (Hussein, et al., 1990; Lima, et al., 1997; Muir, et al., 1990; Potchanakorn y Potter, 1987; Potter, et al., 1995), al no cumplir los requerimientos nutricionales y presentar baja biodisponibilidad de $\mathrm{P}$ en las aves.

Por otra parte, la BRP expresada en PC de la dieta con fosfato dicálcico B fue de 93\% a los $18 \mathrm{~d}$ incrementándose a 100,4\% a los 28 d de edad. Mientras que la BRP como PC de la dieta de fosfato monocálcico C con 100,32\% a los 18 d de vida reduciéndose a 96,84\% a los 28 d de edad (TABLA III). 
Tabla III. Biodisponibilidad relativa (\%) de las diferentes fuentes de fosfato comerciales según los criterios de respuesta en pollos de engorde

\begin{tabular}{lcccc}
\hline \multirow{2}{*}{ Fosfato } & \multicolumn{2}{c}{ PESO } & Cenizas & \\
\cline { 2 - 3 } & 18 Días & 28 Días & 28 Días & MEDIA \pm DE \\
\hline $\begin{array}{l}\text { Monocálcico } \\
(A) *\end{array}$ & 100 & 100 & 100 & 100 \\
Dicálcico (B) & 93 & 100,04 & 117,15 & $103,4 \pm 12,42$ \\
Monocálcio (C) & 100,32 & 96,84 & 91,2 & $96,12 \pm 4,60$ \\
Tricálcico (D) & 88,29 & 88,79 & 65,56 & $80,88 \pm 13,27$ \\
\hline
\end{tabular}

BIOFOS $*=100 \%$ biodisponibilidad del P. $\mathrm{DE}=$ Desviación estándar. Fosfato monocálcico $(\mathrm{A})=$ Biofos comercial. Fosfato dicálcico $(\mathrm{B})=$ Fosbic comercial. Fosfato monocálcico $(\mathrm{C})=$ Monómero. Fosfato tricálcico defluorinado (D) = Tricalfos comercial.

Por otro lado, BRP medido por el PC en los PE fue similar (88,29\% y 88,79) en los d 18 y 28 de vida, respectivamente. La caracterización de las tendencias de la BRP de las fuentes de fosfato y niveles de P representadas como regresiones lineales simples a los 18 y $28 \mathrm{~d}$ de PE se observa en la FIG. 1. 
Figura 1. Relación entre la proporción de inclusión de $\mathrm{P}$ y peso corporal de los pollos de engorde alimentados con diferentes fuentes de $\mathrm{P}$ a los 18 (a) y 28 (b) días de vida
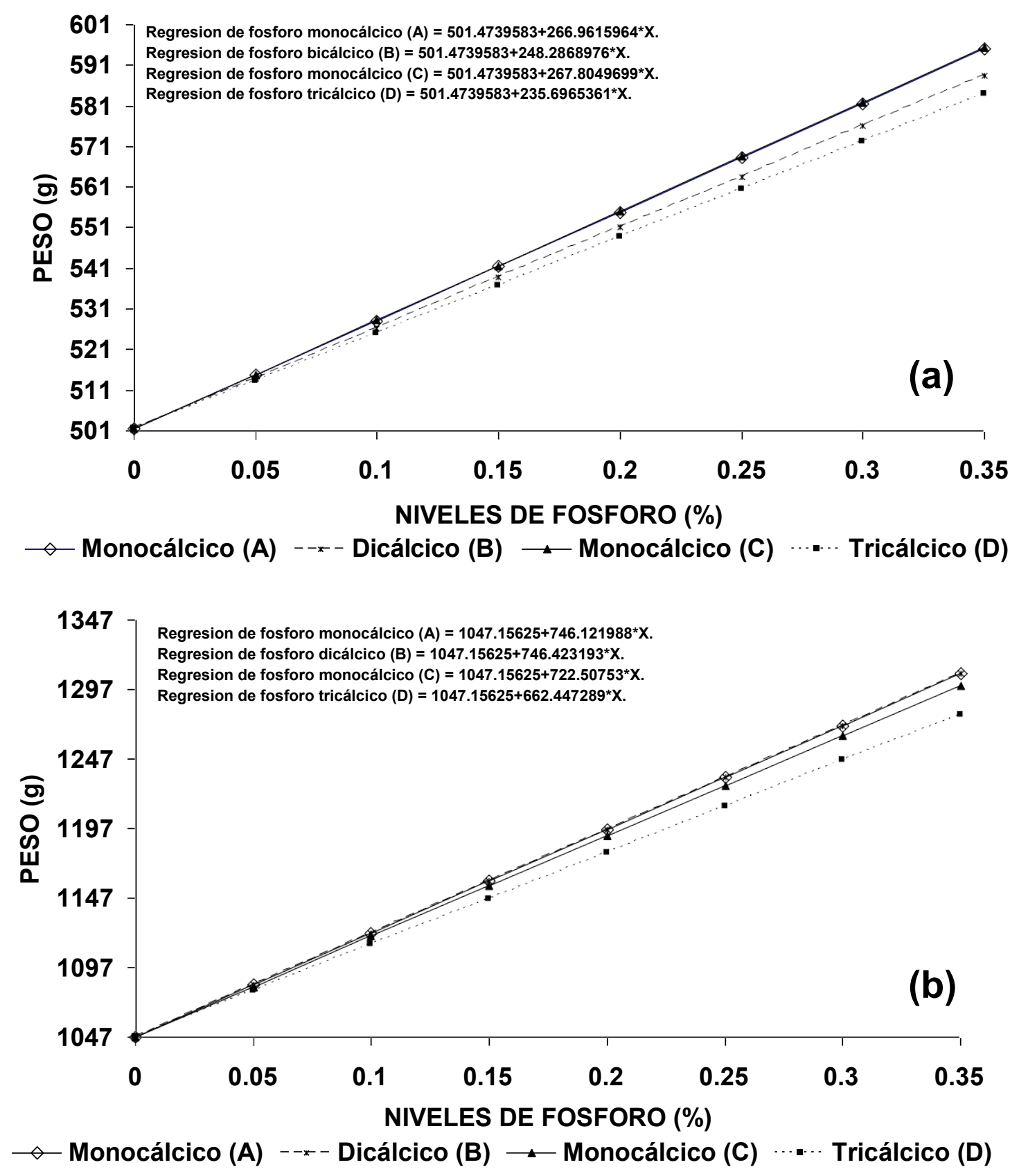
Fosfato monocálcico $(\mathrm{A})=$ Biosfos comercial. Fosfato dicálcico $(\mathrm{B})=$ Fosbic comercial .

Fosfato monocálcico $(\mathrm{C})=$ Monómero. Fosfato tricálcico defluorinado $(\mathrm{D})=$ Tricalfos comercial.

Fuente: Elaboración propia

Estos resultados de la BRP representado por el PC, fueron soportados con el estudio que utiliza como patrón el fosfato dicálcico como mejor comportamiento de la BRP en las aves (Godoy y Chicco, 1999).

En lo referente al PCOT a los $18 \mathrm{~d}$ de edad de los PE, se determinó que el fosfato dicálcico B $(50,12 \pm 1,15 \mathrm{mg} \%)$, monocálcico C $(49,37 \pm 1,33 \mathrm{mg} \%)$ y monocálcico A $(47,79 \pm 1,14 \mathrm{mg} \%)$ fueron los que mejoraron significativamente $(\mathrm{P}<0,001)$ la acreción ósea con el valor más alto de PCOT en contraste con el fosfato tricálcico D $(45,10 \pm 0,86 \mathrm{mg} \%)$ que fue el valor más bajo de PCOT (TABLA IV), aunque el fosfato monocálcico A, fue similar a los demás fuentes de fosfato. Por otro lado, a los $28 \mathrm{~d}$ de edad de las aves el fosfato dicálcico B $(51,83 \pm 1,28 \mathrm{mg} \%)$, monocálcico A $(49,99 \pm 0,80 \mathrm{~g})$ y monocálcico C $(49,62 \pm 0,85 \mathrm{mg} \%)$ fueron los que mejoraron significativamente $(\mathrm{P}<0,001)$ el depósito de minerales en el hueso con el valor más alto de PCOT, en contraste con el fosfato tricálcico D $(46,66 \pm 1,53 \mathrm{mg} \%)$ que fue el valor más bajo de PCOT, mientras que el valor de PCOT del fosfato monocálcico $\mathrm{C}$ fue similar a los demás fosfatos. 
Tabla IV. Porcentaje (\%) de cenizas óseas de tibia derecha en base libre de grasa de pollos de engorde alimentados con diferentes fuentes y niveles de $\mathrm{P}$ a los 18 y 28 días de edad. Valores expresados en media \pm error estándar. ANOVA

\begin{tabular}{llcccc}
\hline Efecto & \multicolumn{1}{c}{ Fosfato } & N & 18 Días (mg\%) & N & 28 Días (mg\%) \\
& Monocálcico (A) & 48 & $47,79 \pm 1,14 \mathrm{ab}$ & 43 & $49,99 \pm 0,80 \mathrm{a}$ \\
& Dicálcico (B) & 48 & $50,12 \pm 1,15 \mathrm{a}^{*}$ & 48 & $51,83 \pm 1,28 \mathrm{a} *$ \\
& Monocálcico (C) & 48 & $49,37 \pm 1,33 \mathrm{a}$ & 48 & $49,62 \pm 0,85 \mathrm{ab}$ \\
& Tricálcico (D) & 48 & $45,10 \pm 0,86 \mathrm{~b}$ & 44 & $46,66 \pm 1,53 \mathrm{~b}$ \\
& 0,15 & 64 & $48,67 \pm 0,77 \mathrm{a}$ & 60 & $45,95 \pm 1,18 \mathrm{~b}$ \\
$\begin{array}{l}\text { Niveles de P } \\
\text { (\%) }\end{array}$ & 0,25 & 64 & $50,82 \pm 0,91 \mathrm{a} * * *$ & 64 & $51,56 \pm 1,08 \mathrm{a} * * * *$ \\
& 0,35 & 64 & $45,42 \pm 1,15 \mathrm{~b}$ & 59 & $51,11 \pm 0,47 \mathrm{a}$ \\
& & & & & \\
\hline
\end{tabular}

Fosfato monocálcico $(\mathrm{A})=$ Biosfos comercial. Fosfato dicálcico $(\mathrm{B})=$ Fosbic comercial. Fosfato monocálcico $(\mathrm{C})$ = Monómero. Fosfato tricálcico defluorinado (D) = Tricalfos comercial. $(\mathrm{a}, \mathrm{b})$ Diferencia estadística significativa $*(\mathrm{P}<0,05) . * * *(\mathrm{P}<0,001) . * * * *(\mathrm{P}<0,0001)$.

Fuente: Elaboración propia

Estos resultados fueron soportados por los hallazgos de los reportes relativos al criterio del PCOT, fue más sensible para medir BRP en las aves, en el caso del PCOT fue influenciado por la fuente de fosfato (Ravindran, et al., 1995), coinciden con la peor acreción ósea fue producida por los fosfatos tricálcicos (Lima, et al., 1999), mientras el fosfato dicálcico utilizado como patrón en otras investigaciones presento el mejor comportamiento en el PCOT (Godoy y Chicco, 1999; Lima, et al., 1997), en contraste con otro estudio no presentaron diferencia por fuente de fosfato (Godoy y Chicco, 1999).

Cuando se analizaron los resultados controversiales del PCOT, por el nivel de suministro de P en las dietas a los 18 d de edad en los $\mathrm{PE}$ mejora significativamente $(\mathrm{P}<0,001)$ la deposición de minerales

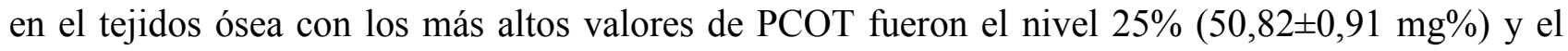
$0,15 \%$ de $\mathrm{P}(48,67 \pm 0,77 \mathrm{mg} \%)$ con el más bajo valor $(45,42 \pm 1,15 \mathrm{mg} \%)$ el nivel de $0,35 \%$. El 
comportamiento más acorde de los resultados del PCOT, fueron a los $28 \mathrm{~d}$ de edad de las aves, según el nivel de suplementación de $\mathrm{P}$ en la dieta efecto significativamente $(\mathrm{P}<0,0001)$ sobre el depósito de minerales óseos, con los más altos valores de PCOT fueron el nivel 25\% de P $(51,56 \pm 1,08 \mathrm{mg} \%)$ y $0,35 \%$ de $\mathrm{P}(51,11 \pm 0,47 \mathrm{mg} \%)$ con el más bajo valor el nivel de $0,15 \%$ de $\mathrm{P}(45,95 \pm 1,18 \mathrm{mg} \%)$. Estudios referentes a las concentraciones de P en la dieta expresan que el PCOT se incrementa con el nivel de P (Driver, et al., 2005), estos resultados fueron contradictorios al no detectar diferencias en otras investigaciones (Godoy y Chicco, 1999).

Los resultados del efecto de las dietas experimentales según la fuente de fosfato y nivel aportado de P sobre el PCOT de los PE 18 y 28 d de edad. La dieta del tratamiento 8 (fosfato monocálcico C y $0,25 \%$ de $\mathrm{P})$ mejoró significativamente $(\mathrm{P}<0,01)$ a los $18 \mathrm{~d}$ de vida de las aves obteniendo el valor más alto $(54,43 \pm 2,18 \mathrm{mg} \%)$ de PCOT y el valor $(41,91 \pm 1,41 \mathrm{mg} \%)$ más bajo de PCOT de la dieta del tratamiento 12 (fosfato tricálcico D y $0,35 \%$ de P). El PCOT afectado por la dieta del tratamiento 8 fue estadísticamente $(\mathrm{P}<0,0001)$ diferente a los tratamientos 3, 6, 7, 9, 10, 11 y 12 a los $18 \mathrm{~d}$ de edad de las aves. Asimismo a los $18 \mathrm{~d}$ de edad de los PE, el PCOT de la dieta del tratamiento 12 mostró diferencia estadística significativa $(\mathrm{P}<0,0001)$ al confrontarlo con tratamiento 2, 4, 5, 6, 7, 8, 10 y 11 .

Mientras que el PCOT a los 28 d de vida de los PE alimentados con la dieta del tratamiento 6 (fosfato dicálcico $\mathrm{B}$ y $0,35 \%$ de $\mathrm{P})$ mejoró significativamente $(\mathrm{P}<0,0001)$ con el valor más alto $(52,71 \pm 0,59$ $\mathrm{mg} \%$ ) y el valor medio $(36,84 \pm 4,39 \mathrm{mg} \%$ ) más bajo de PCOT fue la dieta del tratamiento 10 (fosfato tricálcico D y $0,15 \%$ de P). De esta misma forma la dieta del tratamiento 10 el valor medio del PCOT fue estadísticamente diferente $(\mathrm{P}<0,0001)$ a los valores de medios del PCOT de las dietas de los tratamientos $1,2,3,4,5,6,7,8,9,11$ y 12 a los $28 \mathrm{~d}$ de vida de las aves.

En el valor medio de PCOT de la dieta del tratamiento 6, se observó diferencia estadística significativa $(\mathrm{P}<0,0001)$ al compararlo con los valores medios de PCOT de las dietas de los tratamiento 1, 7 y 10 a los 28 días de edad de las aves. Mientras que el valor medio $(46,59 \pm 0,92 \mathrm{mg} \%)$ de PCOT afectado por la dieta del tratamiento 7 (fosfato monocálcico $\mathrm{C}$ y $0,15 \%$ de P) presentó diferencia estadística significativa $(\mathrm{P}<0,0001)$ a los $28 \mathrm{~d}$ de edad de las aves al compararlo con los valores medios del PCOT de las dietas de los tratamientos 5, 6, 8 y 10 (TABLA V). 
Tabla V. Porcentaje de cenizas óseas de tibia derecha en base libre de grasa de pollos de engorde alimentados con tratamientos de diferentes fuentes y niveles de $\mathrm{P}$ a los 18 y 28 días de edad.

Valores expresados en media \pm error estándar. ANOVA

\begin{tabular}{|c|c|c|c|c|c|c|}
\hline \multirow{2}{*}{ Tratamiento } & \multirow{2}{*}{ Fuente } & \multirow[b]{2}{*}{$\%$} & \multicolumn{4}{|c|}{ Edad } \\
\hline & & & $\mathrm{N}$ & 18 Días (mg\%) & $\mathrm{N}$ & 28 Días $(\mathrm{mg} \%)$ \\
\hline 1 & & 0,15 & 16 & $49,62 \pm 0,97 \mathrm{ab}$ & 16 & $47,51 \pm 0,85 \mathrm{bc}$ \\
\hline 2 & Monocálcico (A) & 0,25 & 15 & $49,32 \pm 2,31 \mathrm{abc}$ & 16 & $51,55 \pm 1,78 \mathrm{abc}$ \\
\hline 3 & & 0,35 & 15 & $44,30 \pm 2,24 \mathrm{~cd}$ & 11 & $51,33 \pm 0,80 \mathrm{abc}$ \\
\hline 4 & & 0,15 & 16 & $50,75 \pm 1,87 \mathrm{ab}$ & 16 & $50,57 \pm 1,56 a b c$ \\
\hline 5 & Dicálcico (B) & 0,25 & 16 & $50,49 \pm 1,48 \mathrm{ab}$ & 16 & $52,20 \pm 3,51 \mathrm{ab}$ \\
\hline 6 & & 0,35 & 16 & $49,12 \pm 2,57 \mathrm{bc}$ & 16 & $52,71 \pm 0,59 \mathrm{a} * * * *$ \\
\hline 7 & & 0,15 & 16 & $47,19 \pm 1,52 b c$ & 16 & $46,59 \pm 0,92 \mathrm{c}$ \\
\hline 8 & Monocálcico (C) & 0,25 & 16 & $54,43 \pm 2,18 \mathrm{a} * *$ & 16 & $52,19 \pm 1,88 \mathrm{ab}$ \\
\hline 9 & & 0,35 & 15 & $46,32 \pm 2,66 \mathrm{bcd}$ & 16 & $50,09 \pm 1,17 \mathrm{abc}$ \\
\hline 10 & & 0,15 & 16 & $47,11 \pm 1,61 b c$ & 12 & $36,84 \pm 4,39 d$ \\
\hline 11 & Tricálcico (D) & 0,25 & 16 & $48,96 \pm 0,77 b c$ & 16 & $50,30 \pm 0,62 \mathrm{abc}$ \\
\hline 12 & & 0,35 & 16 & $41,91 \pm 1,41 d$ & 16 & $50,38 \pm 0,94 a b c$ \\
\hline
\end{tabular}

Fosfato monocálcico $(\mathrm{A})=$ Biosfos comercial. Fosfato dicálcico $(\mathrm{B})=$ Fosbic comercial. Fosfato monocálcico $(\mathrm{C})$ = Monómero. Fosfato tricálcico defluorinado $(\mathrm{D})=$ Tricalfos comercial. $(\mathrm{a}, \mathrm{b}, \mathrm{c}, \mathrm{d})$ $(\mathrm{a}, \mathrm{b}, \mathrm{c})$ Diferencia estadística significativa. $* *(\mathrm{P}<0,01) . * * * *(\mathrm{P}<0,01)$.

Fuente: Elaboración propia

$\mathrm{Al}$ analizar los interceptos de la pendiente de regresiones, por fuente de fosfato utilizando el PCOT como criterio de respuesta para la BRP a los 28 d de vida de las aves, se detectó el efecto de la dieta 
del fosfato dicálcico B (117,15\%) fue muy superior a la dieta patrón del fosfato A $(100 \%)$ y otras dietas de fosfato C $(91,20 \%)$ y D $(65,56 \%)$, respectivamente (FIG. 2 y TABLA III).

Figura 2. Relación entre el porcentaje de inclusión de P y ceniza ósea de las aves alimentadas con diferentes fuentes de fosfato hasta los 28 días de edad

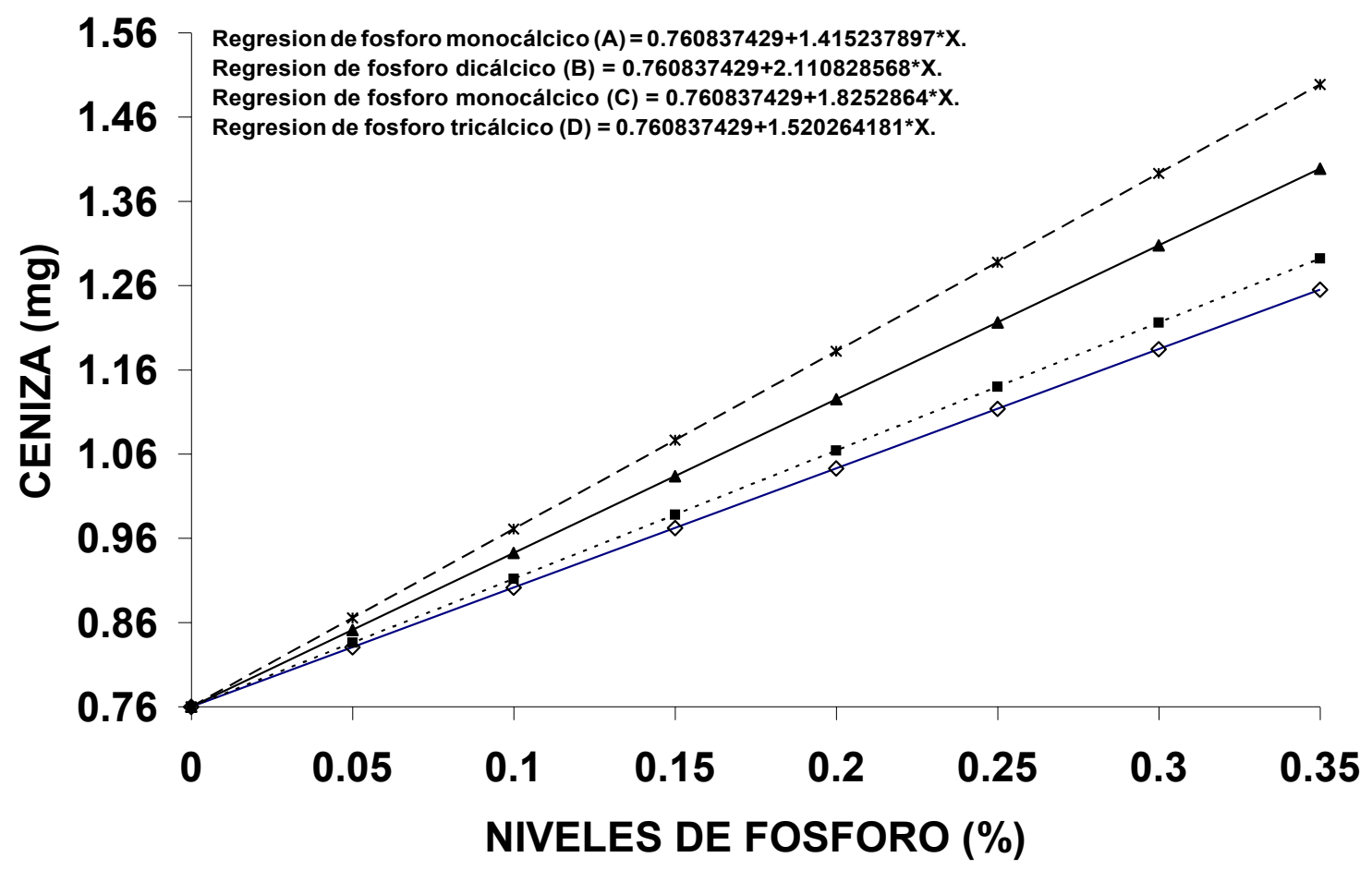

$\multimap$ Monocálcico (A) -*- Dicálcico (B) —-Monocálcico (C) … * Tricálcico (D)

Fosfato monocálcico $(\mathrm{A})=$ Biosfos comercial. Fosfato dicálcicos $(\mathrm{B})=$ Fosbic comercial. Fosfato monocálcico $(\mathrm{C})=$ Monómero. Fosfato tricálcico defluorinado $(\mathrm{D})=$ Tricalfos comercial.

Fuente: Elaboración propia

Reportes contradictorios referentes a la BRP de PCOT fueron con fosfato dicálcico y monocálcico presento rangos más cercanos entre sí (Lima, et al., 1997) y detectó o fuentes de fosfato dicálcico fue superior a todas las demás fuentes (Godoy y Chicco, 1999). Se detectó una relación entre el PC y PCOT $28 \mathrm{~d}$ de edad presento una correlación moderada y positiva de $\mathrm{r}=0,58(\mathrm{P}<0,0001)$ y otras 
investigaciones obtuvieron coeficiente de correlación superior al de esta investigación (Godoy y Chicco, 1999).

La respuesta biológica de las aves a la dieta de fosfato dicálcico B, puede ser debido a la acción de las enzima fosfatasa alcalina intestinal permite la absorción del fosfato orgánico no fitato, no orgánico incorporados como el fosfato dicálcico en la dieta (Galvanovskii, et al., 1985), lo que permite fijación de mayor cantidad de fosfato en forma de cristales de hidroxiapatita en el hueso incrementado la acreción ósea y lo cual se refleja en un PCOT y se perceptible mejor a los $28 \mathrm{~d}$ de vida de las aves. Lo cual lo confirma por el aumentaba la inclusión del fosfato aumentaba el porcentaje de ceniza ósea hasta cierto punto, y a partir del cual no se determinó incrementos de la ceniza ósea de tibia al aumentar el porcentaje de inclusión de fosfato en la dieta (Godoy, et al., 2007).

El análisis de la información del PM a los 18 y 28 d de la fuente de fosfato, nivel de $\mathrm{P}$ y los tratamientos, no se presentaron diferencia estadística significativa $(\mathrm{P}>0,05)$ en el PM. La media de la PM acumulado a los 18 y 28 días de vida de las aves fueron de $3.02 \pm 0.71 \%$ y $3.33 \pm 0.75 \%$, respectivamente. Estos hallazgos son soportados por investigaciones realizadas en área (Burnell, et al., 1990; Lima, et al., 1997) y contrarios a otros reportes (Potchanakorn y Potter, 1987; Potter, et al., 1995).

\section{Conclusiones}

A medida que se incrementa el porcentaje de inclusión de $\mathrm{P}$ en las dietas experimentales, aumenta al PC de los PE de 18 y 28 d de edad, en especial la dieta de fosfato monocálcico A con 0,35\% de P a los 18 y 28 d de edad. Se determinó que hay un efecto de la fuente y el nivel de inclusión de $\mathrm{P}$ en las dietas sobre el PCOT derecha de los PE a los 18 y 28 d de edad, considerándose la mejor dieta del fosfato dicálcico $\mathrm{B}$ con $0,35 \%$ de $\mathrm{P}$ a los $28 \mathrm{~d}$ de vida. Los tratamientos, las fuentes y niveles de inclusión de P en las dietas experimentales no afectaron el PM de los PE a los 18 y 28 d de edad. Se determinó que existe una relación entre los criterios de BRP entre el PC y la PCOT de los PE a la edad de $28 \mathrm{~d}$. 


\section{Referencias Bibliográficas}

A.O.A.C. 1984. Official methods of analysis. 15th ed. Association of Official Analytical Chemists. Washington, D. C. USA. $1081 \mathrm{p}$

Akpe, M. P., P. E. Waibel, K. Larntz, A. L. Metz, S. L. Noll and M. M. Walser. 1987. Phosphorus availability bioassay using bone ash and bone densitometry as response criteria. Poult Sci. 66(4):71320

Arias, A., S. Godoy, P. Pizzani and C. Chicco. 2008. Validaton of productive response and bone mineralization in chicken fed with urea phosphate (up) in the feed and water. Rev. Cient. 18(4):40814

Askelson, T. E., A. Campasino, J. T. Lee and T. Duong. 2014. Evaluation of phytate-degrading Lactobacillus culture administration to broiler chickens. Appl Environ Microbiol. 80(3):943-50

Burnell, T. W., G. L. Cromwell and T. S. Stahly. 1990. Effects of particle size on the biological availability of calcium and phosphorus in defluorinated phosphate for chicks. Poult Sci. 69(7):11107

Coon, C. N., S. Seo and M. K. Manangi. 2007. The Determination of Retainable Phosphorus, Relative Biological Availability, and Relative Biological Value of Phosphorus Sources for Broilers. Poult Sci. 86(5):857-68

COPLANARH, (Comisión del Plan Nacional de Aprovechamiento de los Recursos Hidráulicos). 1975. Atlas Inventario Nacional de Tierras. Región Lago de Maracaibo. Tecnicolor S. A. Caracas. Venezuela. $275 \mathrm{p}$

Davies, M. I. and I. Motzok. 1972. Properties of chick intestinal phytase. Poult Sci. 51(2):494-501

Driver, J. P., G. M. Pesti, R. I. Bakalli and H. M. Edwards, Jr. 2005. Effects of calcium and nonphytate phosphorus concentrations on phytase efficacy in broiler chicks. Poult Sci. 84(9):1406-17

Fernandes, J. I., F. R. Lima, C. X. Mendonca, Jr., I. Mabe, R. Albuquerque and P. M. Leal. 1999. Relative bioavailability of phosphorus in feed and agricultural phosphates for poultry. Poult Sci. 78(12):1729-36 
Fick, K.R., S.M. Miller, J.D.; Funk, L.R. McDowell and R.H. Houser. 1979. Methods of mineral analysis for plant and animal tissues. Animal Science Departament. Universidad de Florida. Gainesville, Florida. 502 p

Fuenmayor, W. 2005. Atlas del Estado Zulia. Síntesis Socio Histórico Cultural y Geográfica. Edición Especial y Actualizada. Quinta Edición. Editado por Splanos C. A. Maracaibo. Venezuela. 181 p

Gabel, N. W. and V. Thomas. 1976. Inorganic polyphosphate as an integral part of alkaline phosphatase preparations. Bioinorg Chem. 5(3):189-97

Galvanovskii, IuIa, MIu Valinietse and V. K. Bauman. 1985. [Effect of vitamin D on inorganic phosphorus transport and alkaline phosphatase activity of the small intestine of chickens]. Fiziol $\mathrm{Zh}$ SSSR Im I M Sechenova. 71(2):243-7

Gillis, M. B., L. C. Norris and G. F. Heuser. 1954. Studies on the biological value of inorganic phosphates. J Nutr. 52(1):115-25

Godoy, S. and C.F. Chicco. 2005. Retención y cinética del fósforo en pollos de engorde alimentados con fosfatos sedimentarios. Interciencia. 30(4):217-20

Godoy, S. and C. Chicco. 1999. Fosfatos Sedimentarios Venezolanos en la Nutrición de Pollos de Engorde. I. Crecimiento y Mineralización del Tejido Óseo. Revista Científica, FCV-LUZ. 9(4):28291

Godoy, S. and C. Chicco. 1999. Fosfatos Sedimentarios Venezolanos en la Nutrición de Pollos de Engorde. II. Efecto del Flúor. Revista Científica, FCV-LUZ. 9(5):372-77

Godoy, S., C. Chicco, A. Morgado, P. Pizzani, A. Arias and J. Palma. 2007. Crecimiento y mineralización ósea de pollos de engorde alimentados con fosfatos nacionales. Zootecnia Trop. 25(4):291-99

Godoy, S. and C.F. Chicco. 2001. Relative bio-availability of phosphorus from Venezuelan raw rock phosphates for poultry. Animal Feed Science and Technology. 94(3-4):103-13

Hemati, H.R., F. Dashtbin and J. Salari. 2013. Absorption and Macromineral Interactions in Broiler Production: An Overview. Global Veterinaria. 11(1):49-54 
Hughes, A. L., J. P. Dahiya, C. L. Wyatt and H. L. Classen. 2009. Effect of Quantum phytase on nutrient digestibility and bone ash in White Leghorn laying hens fed corn-soybean meal-based diets. Poult Sci. 88(6):1191-8

Hussein, A. S., A. H. Cantor, T. H. Johnson and R. A. Yokel. 1990. Effect of dietary aluminum sulfate on calcium and phosphorus metabolism of broiler chicks. Poult Sci. 69(6):985-91

Hussein, A. S., A. H. Cantor, A. J. Pescatore and T. H. Johnson. 1993. Effect of dietary aluminum and vitamin D interaction on growth and calcium and phosphorus metabolism of broiler chicks. Poult Sci. 72(2):306-9

Kim, E. J., C. M. Amezcua, P. L. Utterback and C. M. Parsons. 2008. Phosphorus bioavailability, true metabolizable energy, and amino acid digestibilities of high protein corn distillers dried grains and dehydrated corn germ. Poult Sci. 87(4):700-5

Kozlowski, K., J. Jankowski and H. Jeroch. 2009. Efficacy of different phytase preparations in broiler rations. Pol J Vet Sci. 12(3):389-93

Kunitz, M. 1960. Chicken intestinal alkaline phosphatase. I. The kinetics and thermodynamics of reversible inactivation. 2. Reactivation by zinc ions. J Gen Physiol. 43:1149-69

Lima, F. R., J. I. Fernandes, E. Oliveira, G. C. Fronzaglia and H. Kahn. 1999. Laboratory evaluations of feed-grade and agricultural-grade phosphates. Poult Sci. 78(12):1717-28

Lima, F.R., C.X. Mendonça, J.C. Alvarez, J.M. Garzillo, E. Ghion and P.M. Leal. 1997. Biological evaluations of commercial dicalcium phosphates as sources of available phosphorus for broiler chicks. Poult Sci. 76(12):1707-13

Littell, R. C., P. R. Henry, A. J. Lewis and C. B. Ammerman. 1997. Estimation of relative bioavailability of nutrients using SAS procedures. J Anim Sci. 75(10):2672-83

Martinez Amezcua, C., C. M. Parsons and S. L. Noll. 2004. Content and relative bioavailability of phosphorus in distillers dried grains with solubles in chicks. Poult Sci. 83(6):971-6

Muir, F., R.M. Leach and B.S. Heinrichs. 1990. Bioavailability of Phosphorus from Broiler Litter Ash for Chicks. Poultry Science. 69(11):1845-50 
N.R.C. 1994. Nutrient requirements of poultry. National Research Council. 9th ed. Nat. Acad. Press. Washington, D.C. $157 \mathrm{p}$

Peterlik, M. and R. H. Wasserman. 1980. Regulation by vitamin D of intestinal phosphate absorption. Horm Metab Res. 12(5):216-9

Potchanakorn, M. and L. M. Potter. 1987. Biological values of phosphorus from various sources for young turkeys. Poult Sci. 66(3):505-13

Potter, L.M., M. Potchanakorn, V. Ravindran and E.T. Kornegay. 1995. Bioavailability of phosphorus in various phosphate sources using body weight and toe ash as response criteria. Poult Sci. 74(5):81320

Rath, N. C., G. R. Huff, W. E. Huff and J. M. Balog. 2000. Factors regulating bone maturity and strength in poultry. Poult Sci. 79(7):1024-32

Ravindran, V., E. T. Kornegay, L. M. Potter, B. O. Ogunabameru, M. K. Welten, J. H. Wilson and M. Potchanakorn. 1995. An evaluation of various response criteria in assessing biological availability of phosphorus for broilers. Poult Sci. 74(11):1820-30

S.A.S. 2013. SAS Institute Inc. Base SAS ${ }^{\circledR} 9.4$ Procedures Guide: Statistical Procedures. Second Edition. SAS Institute Inc. Cary, NC. 649 p

S.A.S. 2014. Statistical Analysis System. Institute Inc. System SAS for Windows. Versión 9.4. SAS Institute Inc. Cary, NC, USA

Steinsberger, S. C., J. F. Ort and J. C. Shih. 1987. Composition and phosphorus bioavailability of a solid by-product from anaerobically digested waste from caged layer hens. Poult Sci. 66(4):634-9

Valinietse, M. Y., V. K. Bauman and Y. Y. Galvanovsky. 1985. Absence of alkaline phosphataselinked inorganic phosphate transport in the chick jejunum. Digestion. 31(4):182-8

Waibel, P. E., N. A. Nahorniak, H. E. Dziuk, M. M. Walser and W. G. Olson. 1984. Bioavailability of phosphorus in commercial phosphate supplements for turkeys. Poult Sci. 63(4):730-7 